it is the primum mobule in relation to the ventricies on the action of which, and not on that of the aurioles, the maintenance of the corculation and proservation of the life of the organism chefly depend.

The practical point which a study of our cases suggests is, that a man does not die from ventricular failure becanse cardiac motion from the veins and auricles towards the ventricles is blocked or prevented from reaching the latter with normal ease and regularity, but because the independent motile power of the ventricle has been abolished, by the failure on its own part to produce the stimulus to contraction in a sufficient mass of its substance, and with sufficient regularity, to maintain its effective contraction. The regulation of this property of cardiac muscle and of every other manifestation of its life, should surely, like the regulation of all other functions, depend upon the visceral nervous system and its central sources. Indeed is it not admitted to do so? The detached organ has detached with it all its constituents and the artificial circulation which keeps alive muscle for a time after evisceration must also afford pabulum and stimulus to the nervous system of the detached viscus, which, however, according to the present views of some physiologists, is incapable of influencing the cardiac muscle under these circumstances.

But the myogenist rests himself greatly on His's observation that the tube-heart of the embryo pulsates prior to its innervation. Does it do so prior to the genesis of the central nervous system? No. This being so, we cannot tell what the relation of the embryonic centre to the periphery is. There may be an influencing from a distance, so to speak, for ought we know or can know to the contrary. That is, the growing nervous system may influence rhythmicity in the growing cell, even in the absence of actual anatomical connexion between the central and peripheral visceral nervous system. What determines the direction in which the nerves grow into an organ? The question cannot, of course, be answered, but it is not unreasonable to suppose that current from the centre to the cell station, without definite structure, may precede visible organisation.

The general conclusions, then, which a study of our cases suggests is, that man lives on his ventricles and chiefly on his left ventricle, not upon his veins or auricles, and that the normal action of the ventricles is dependent upon the healthy action and interaction of the three fundamental factors in organic life, blood, cell, and nerves, a trinity in which the blood supplies the force necessary to the production and continuance of vital action; in which the cell is the specific agency or mechanism for a given organic act, and in which the nerves regulate alike the production of the motive force and the degree and regularity of its manifestation in the specific cell.

A corollary to this conclusion is, that as man lives on his ventricle, not on his auricle, the examination and registration of ventricular action are of greater practical importance than a similar investigation of the action of his veins and auricles, although as a detail these points have a scientific interest, as showing whether the preventricular and nonlethal portion of the muscular circulatory mechanism is quiescent or tumultuous, extinguished, in abeyance, or in action, any of which it apparently may be, without the death of the organism, if the conclusions drawn from the study of our cases be correct. Even, however, if this conclusion be in the main correct, and doubtless some will not concede this, does it follow that all the careful and exact scientific and clinical observation of the preventricular mechanism is "love's labour lost" from the point of view of practical utility? By no means. For, "facts are chiels that winna .ding and daurna be disputed," and it is a fact that an attenuated muscular connexion of peculiar character, of the existence of which we were not aware until His demonstrated it, does pass from the right auricle to the ventricles. Moreover, it is apparently also a fact that the peculiar structure has a widespread distribution as shown by Tawara, and finally that clamping and cutting the chain before it passes into the ventricles modify the relative actions of the auricles and ventricles. Of these facts the most dubious of interpretation is the last. The question, as I have said, is, What is clamped and cut? Is it muscle only? To this I think the answer is in the negative, and here the myogenist and neurogenist part company. Possibly, and even probably, both are in a measure correct in their views, and wholly so neither.

\section{NASO-ANTRAL POLYPUS.}

BY A. BROWN KELLY, M.D., D.Sc. GLASG., SURGEON FOR DISEASES OF THE THROAT AND NOSE, VICTORIA INFIKMARY, GLASGOW.

THose who have seen many cases of nasal polypus must occasionally have met with the variety which has been termed naso-pharyngeal or choanal polypus. This neoplasm presents several distinctive features. The patient in many instances is a child. The neoplasm is single. If small, it is situated in the posterior part of the nasal fossa; if large, it projects into the naso-pharynx and may even hang below the palate. A lobe or prolongation may extend forward in the nose and reach the anterior naris, so that the case may look like an ordinary one of nasal polypus. In spite of the unilateral character of the growth there is, as a rule, no associated discharge of pus. Removal through the mouth will probably suggest itself as most feasible when the polypus is large. If caught firmly it can be torn away entirely, so that on examining the naso-pharynx not only is no trace of the growth seen but even the bleeding point of attachment is not discoverable. A large cyst is usually present in the polypus. Recurrence is not uncommon. With such well-marked characteristics it is surprising that these growths failed to excite interest and that their antral origin escaped detection until pointed out by Professor G. Killian ${ }^{1}$ about three years ago.

Since the publication of Killian's paper I have had 15 cases of naso-antral polypus. In 11 of these the antrum was opened and the connexion of the lining membrane of the cavity with the growth was demonstrated. In the remaining four cases the conditions were such as to put almost beyond question an antral origin. I shall briefly describe some of the more prominent features observed and the treatment employed in these cases.

Age and sex.-A striking peculiarity and one that distinguishes this class of polypus from the common or ethmoidal type is the patient's age. Of 100 consecutive cases that I have noted of ordinary polypus uncomplicated by accessory cavity suppuration, in only three was the age under 20 namely, 16, 17, and 19 years. On the other hand, of my 15 patients with naso-antral polypus 10 were under 20 years of age. The youngest was 5 and the others were 9 (two), 10, 12 (two), 15, and 16 (three) years old respectively. The ages of the remaining five were between 23 and 35 years ; two of them had had a polypus removed before the age of 20 . Seven of the patients were males and eight were females.

Onset.-Some of the patients gave a history of the symptoms having become quickly marked. Others within a few weeks of feeling obstructed had seen the polypus at the nasal orifice. In most a large growth was found although symptoms had been present for only two or three months previously. It would therefore appear that the polypus may develop rapidly.

Symptoms.-Two symptoms were almost invariably present -namely, snoring and thick speech. A watery discharge from the affected side was sometimes complained of. On one occasion a quantity of clear fluid ran from a patient's nose after sneezing and the obstruction was relieved for a few days afterwards, a cyst having probably ruptured. Enuresis was troublesome in a boy aged nine years. This symptom had set in six weeks previously, coincidently with very marked nasal obstruction, on the removal of which it disappeared entirely; he had never before been thus afflicted. It is noteworthy that in no instance was pain in the cheek or other symptom of intra-antral pressure complained of.

Appearances in nose and naso-pharynx. - When the growth was small it was indistinguishable from an ordinary polypus situated below the posterior half of the middle turbinate. At a more advanced stage there was much the same appearance anteriorly, but by posterior rhinoscopy the polypus could be seen passing backwards and simulating the hypertrophied posterior end of an inferior turbinate, or, when larger, occupying more or less of the naso-pharynx and even hanging down behind the soft palate. In about half of my cases there was, in addition to the main mass of the polypus just described, a prolongation forward which in several instances reached as far as the anterior naris. I have 
hitherto met with no case in which ethmoidal or other polypi were present in either nasal cavity; the designation solitary is therefore applicable to the naso-antral polypus. A scanty purulent discharge was detected in two cases.

Iransillumination. - This test was tried in nine cases-in eight of which the antrum was subsequently opened-and in all the affected cavity transillumined well. For the sake of brevity particulars as to the crescentic tache, pupil and subjective light sensation in each case are omitted. In two instances the normal side was rather the brighter; these were the cases in which a purulent discharge was observed in the nose; in one of them the antrum was not opened, in the other besides a cyst filling the lumen of the cavity a purulent cyst and thickened lining membrane were found. In three cases transillumination on the two sides was equal; in two of these a cyst occupied the cavity; in the third numerous closely packed polypi were present. In four cases the antrum on the affected side transillumined better than that on the normal side; in all of these a large cyst filled the cavity, and in one instance several polypi of the lining membrane were also present.

From the point of view of the intra-antral condition the results of transillumination may be briefly summarised as follows. In the eight cases in which the test was applied and the antrum subsequently opened a cyst was found filling the cavity in seven instances. In all of these, with one exception, transillumination on the affected side was equal to or better than that on the other side. In the exception noted, besides the cyst there was general thickening of the lining membrane, and transillumination on this side was less bright than on the other. In the eighth case the antrum was almost filled with polypi and transillumination on the two sides was equal.

The fact that an antrum containing one or more cysts or polypi transillumines as well as, or better than, the cavity on the other side, which is presumably normal, has been commented upon by several observers but no explanation has been forthcoming. A dental or maxillary cyst which has invaded the antrum may favour transillumination by causing thinning of the bony walls. No such factor is in force in the case of cysts and polypi of the antral lining membrane, these being delicate growths which are incapable of causing bony absorption.

I might here refer to two other phenomena of transillumination which have seemed inexplicable. The first is that observed after thoroughly removing the thickened lining membrane of an antrum when the cavity, instead of transillumining normally, as one would expect, remains dark. A view which cannot meet with general acceptance has been hazarded that the obscurity is due to changes in the bone. The second phenomenon is that occasionally noticed during an acute non-suppurative antral catarrh, when the cavity transillumines badly. This is usually ascribed to swelling of the lining membrane. If we consider, however, the extreme thinness of the normal antral mucosa and that serous exudation into its substance would interfere little, if at all, with the transmission of light, this view cannot be regarded as satisfactory.

All three anomalnus appearances can be explained, I believe, by taking into account the optical condition within the antrum. In the normal cavity the concave bony walls and the smooth, shining membrane cause a condensation of light and favour transillumination. When catarrhal, the lining membrane becomes velvety, dull, and loses its reflecting power, so that the light entering the antrum is largely absorbed and transillumination is diminished. After the muco-periosteum has been removed the innumerable minute irregularities on the bare bony walls scatter the light passing into the cavity so that transillumination is again less. On the other hand, in the case of a. cyst transillumination is favoured by two factors : first, the decrease of diffuse reflection by the obliteration of the irregularities of those portions of the antral wall with which the sac is in contact; and second, the condensation caused by refraction due to the presence of fluid in the sac. For pointing out the latter factor I am indebted to my friend Dr. A. Freeland Fergus.

In further support of the explanation offered I may mention that I have transillumined patients with naso-antral polypus immediately before operation and have found the affected side the brighter. The antrum has then been opened in the manner described below, and on transillumination being repeated the same result has been obtained. A cyst which filled the antrum has next been removed and on transillumining immediately afterwards this side instead of being the brighter, as previously, has been found to be much the darker. Severai weeks later, on again applying the test both sides have transillumined equally well; the assumption is that by this time the lining membrane had regained its polish which the pressure of the cyst had temporarily destroyed.

Treatment.-I have employed two methods of treatment. The first aims mainly at the removal of the nasal portion of the growth. If small, it is caught directly by a snare; if somewhat larger and hanging into the naso-pharynx, it is drawn into the nose by means of Lange's hook and then caught in the snare ; if very large, it is encircled by a loop passed upwards from the mouth. This last procedure is best accomplished by using a snare tube curved after the fashion of naso-pharyngeal probes or forceps. The wire loop of a suitable size and shape is doubled downwards and backwards over the edge of the cannula; the latter is then passed upwards between the posterior wall of the pharynx and the polypus; on tightening, the loop, if properly adjusted, passes upwards between the soft palate and growth and thus encircles the latter. In whatever manner the polypus is caught it should not be cut through but merely constricted until a good grip is obtained and then torn away. I have thus often removed the intraantral together with the entire nasal portion of the growth the former has consisted, in the great majority of cases, of the whole or a portion of a collapsed cyst. This method is not always satisfactory on account of the frequency of recur rence. In seven of my 15 cases the polypus had been removed at least twice. One boy was operated upon in 1897, 1898, 1899, 1900, and 1907; another in 1898, 1899, 1900 (twice), and radically in 1906; while a girl, between 1900 and 1907, when the radical operation was performed, had a polypus removed seven times. Mr. Richard Lake ${ }^{2}$ has reported a similar case.

Such results led me to adopt the second method of treat. ment. This consists in opening the antrum, determining the intra-antral attachments of the growth, and completely removing these together with the polypus in the nose. The mode I employ of gaining access to the antrum is the same as that previously recommended by me. ${ }^{3}$ The operation is performed under local anæsthesia excepting when the patient is a child. A weak solution of cocaine, or one of its substitutes with a little adrenalin, is injected into the soft tissues over the canine fossa. An incision is made in the gingivo-labial fold, the lower part of the facial wall of the superior maxilla is exposed, and a small opening is bored in this by means of a sharp-pointed trocar and afterwards enlarged by a blunt-pointed trocar. The use of the latter instrument lessens the risk of rupturing an intra-antral cyst when present and of injuring the opposite bony wall. The cavity is then examined by the aid of an antral speculum (illustrations of this and of other instruments used may be seen in the paper just referred to)

In 11 cases thus inspected the following were the intraantral conditions found. A large cyst occupied the entire cavity in seven; the lining membrane was polypoid in two and generally thickened in two. In ten of the cases one large ostium accessorium was present, while in the eleventh there were two separated by a thin fibrous band. The appearances were practically the same in all the cases in which a cyst was found. When the cyst wall had escaped injury from the trocars it bulged into the opening in the bone as a smooth fluctuating membrane. By means of a director the cyst wall was easily separated from the antral lining membrane. In the course of the manipulations the cyst was usually ruptured and collapsed and then its connexion through the accessory opening with the polypus in the nose became apparent. Sometimes, however, while boring into the antrum the cyst was also opened and a considerable amount of fluid escaped. On inspecting the cavity it was then seen to be lined with a moist, pale polypoid tissue which might have readily been mistaken for the altered antra lining membrane; with care, however, it could be stripped from the latter. In two cases one or more small cysts were found in addition on the inner wall near the accessory opening and in one of these the lining membrane was also thickened. In another case several polypi were present in

2 A Case of Cystic Disease of the Maxillary Antrum, THE LixCET, March 14th, 1908, p. 783

${ }^{3}$ Inspection of the Antrum of Highmore, ThE Lancer, Sept. 17th, 1904, p. 819 
the posterior part of the cavity. In the others the lining membrane was normal. In the four instances in which no cyst was present the lumen of the cavity was considerably reduced by general thickening of the lining membrane in two cases and by large flat polypi in two cases. The treatment of these conditions varied as required from curetting the affected areas to the removal of the entire lining membrane.

The intra-antral conditions having been dealt with the polypus in the nose or naso-pharynx is gripped and torn away in the manner already described. By this plan the polypus, together with the collapsed antral cyst, or more or less of the thickened antral lining membrane is obtained. The antrum is again carefully inspected, particularly around the ostium accessorium, and any small cysts or polypoid proliferations that may remain are destroyed. The opening in the gingivolabial fold is allowed to heal up. If there has been much bleeding in the antrum syringing of the cavity is carried out for a few days. Time will show whether these measures are sufficient or whether in a subject with a large ostium accessorium and a lining membrane that has given rise to one or more cysts the removal of the entire lining membrane is not necessary. It should be remembered that antral cysts may be present which are so small as to be visible only on closest examination. ${ }^{4}$ The typical naso-antral polypus after removal is found to have a characteristic appearance. The naso-pharyngeal portion is firm, rounded, sometimes partially bi-lobed, often cystic, and continued directly into the nasal portion which is softer and like an ordinary mucous polypus. The antral portion passes off at right angles to the preceding two; it is delicate, succulent, and consists of a cyst wall.

Pathogenesis.-From a consideration of the foregoing facts it is evident that the choanal or naso-pharyngeal polypus takes its origin in the antrum, and hence is better termed naso-antral polypus. The morbid condition present in the cavity is most frequently a cyst, but it may also be a polypus or general thickening of the lining membrane. A large opening of communication between the antrum and nose is essential. In all of my cases this has been supplied by an ostium accessorium, although it is conceivable that the ostium maxillare might serve the purpose. Through the opening a portion of the cyst, polypus or thickened membrane protrudes and gradually enlarges. Development proceeds in the direction of least resistance-namely, towards the naso-pharynx and pharynx. Owing to the constriction at the ostium there is a tendency to odema of the polypus and to the formation of false cysts in its substance. In a previous communication I pointed out that antral cysts most frequently spring from the inner wall of the cavity and that in subjects with antral cysts an accessory opening occurs four times oftener than usual. ${ }^{5}$ Two of the conditions necessary for the development of the naso-antral polypus are thus commonly found associated.

In conclusion, I might mention that I have also met with recurrent cystic polypi growing down between the septum and posterior part of the middle turbinate. It is highly probable that these originate in the sphenoidal sinus, but so far I have had no case in which I felt warranted in removing sufficient of the ethmoid to determine this point.

Glasgow.

\section{FIVE YEARS' EXPERIENCE WITH DUBOIS'S APPARATUS FOR ADMINISTERING CHLOROFORM.}

BY PALL M. CHAPMAN, M.D. LOND., F.R.C.P. LOND., PHYSICIAN TO THE HEREFORDSHIRE GENERAL HOSPITAL.

I HAVE now had upwards of five years' experience in the induction and maintenance of anæsthesia for surgical operations by means of Dubois's apparatus. I began using it with so much anxious timidity and for the first few years was so conscious of my own inability to speak with authority owing to my cases being neither sufficiently numerous nor sufficiently varied that, beyond calling attention to the fact that the apparatus was in use and possessed certain merits of its own, I have published nothing further, contenting myself with using the apparatus in every possible case and,

4 Dmochowski, Z.: Archiv fun Laryngologie, Band iii., p. 255. 5 A Contribution to the Pathology of the Antrum of Highmore Glasgow Medical Journal, October and November 1904. as far as I could obtain them, in cases of every conceivable difficulty. This has been easier of late, as it is in just these anxious and difficult cases that my services have been more and more required. They now amount to many hundreds and I may claim the hearing due to one with five years' varied and practical experience of his method.

The essential part of the apparatus I need not explain again in detail, as I have described it very fully already. ${ }^{1}$ It is, however, advisable to call attention again and again to certain points. 1. That the mixture of $\mathrm{CHCl}_{3}$ with air is supplied ready made from a small gasometer by propulsion through a mask which has a free outlet. 2. That the method is therefore an open method. 3. The supply of the mixture is continuous and is altogether independent of the inspiratory efforts of the patient. 4. That the percentages of $\mathrm{CHCl}_{3}$ with air are real percentages and do not vary, plus or minus, more than $\frac{1}{10}$ th per cent., as proved by numberless densimetric experiments.

One innovation of my own by which the piston which expels the mixture from the gasometer is kept in motion by a movement of the operator's foot was introduced in the first year, and I have used it ever since. It has worked admirably, and I have never had any wish to change it or thought it desirable to improve it in any way. This innovation I had better describe in some detail as the movement of the foot is so slight that it causes no fatigue to the operator when sitting on a suitable stool. The movement becomes quite automatic with use, thus leaving both hands and the whole attention free during an operation which may last from one to two hours.

Without this addition the piston of Dubois's apparatus is set in motion by a hand crank acting on the upper edge of a cogged wheel. Both crank and wheel are situated to the right of the uprights fixed upon the gasometer where the tube is connected with the face-piece, leading off to the left. The foot-piece must also be to the left, and must be near the level of the floor, while the apparatus must be within reach of the administrator's hand when he wishes to change the percentages of the vapour he is giving. A horizontal shaft is therefore run across the surface of the cylinder from left to right, at right angles to the cogged gear-wheel which moves the piston, and anterior to the piston shaft. This horizontal shaft at its right extremity is furnished with cogs which engage with the cogs of the gear-wheel, as seen in Fig. 1.

Fig. 1.

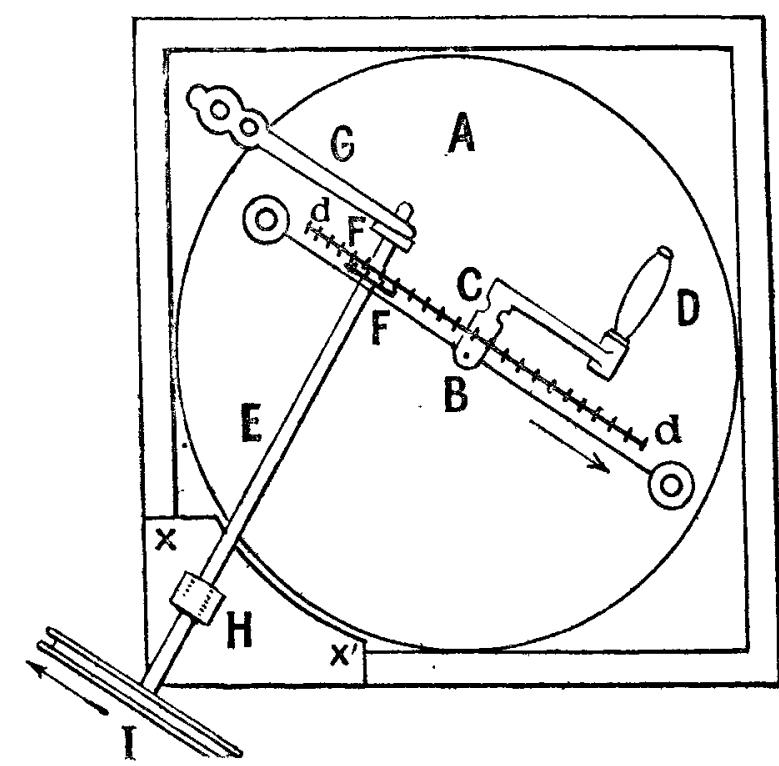

Dubois's apparatus viewed from above. A. Cylindrical gasometer. B. Piston rod, moved by the crank handle $\mathrm{D}$, engaging by pinion the large gear-wheel $d-d$ on its upper edge by cogs at $\mathrm{c}$.

To dispense with using $\mathbf{D}$, and in order to substitute a foot movement, a steel shaft $\mathrm{E}$, carrying cogs at $\mathrm{F}$, is placed under the wheel $d-d$, so that when the shaft $E$ is pushed home into a hole in the clamp $G$ the cogs at $F$ engage on the under edge of the wheel $\mathrm{d}-\mathrm{d}$. The shaft $\mathrm{E}$ runs through a bearing which is fixed upon the corner plate $H$. This plate is detachable and is pinned to the corner of the box at $x$ and $x^{\prime}$ by a thumb-screw at $x^{\prime}$. At its other end, projecting over 